ANNALES

POLONICI MATHEMATICI

$81.2(2003)$

\title{
On extremal holomorphically contractible families
}

\author{
by MAREK JARNiCKI (Kraków), Witold JARNiCKI (Kraków) \\ and Peter Pflug (Oldenburg)
}

\begin{abstract}
We prove (Theorem 1.2) that the category of generalized holomorphically contractible families (Definition 1.1) has maximal and minimal objects. Moreover, we present basic properties of these extremal families.
\end{abstract}

1. Introduction. Main results. First recall the standard definition of a holomorphically contractible family (cf. [Jar-Pfl 1993, §4.1]). A family $\left(d_{G}\right)_{G}$ of functions

$$
d_{G}: G \times G \rightarrow \mathbb{R}_{+}:=[0, \infty),
$$

where $G$ runs over all domains $G \subset \mathbb{C}^{n}$ with arbitrary $n \in \mathbb{N}$, is said to be holomorphically contractible if the following two conditions are satisfied:

- for the unit disc $E$ we have $d_{E}(a, z)=m_{E}(a, z):=\left|\frac{z-a}{1-\bar{a} z}\right|$ for $a, z \in E$,

- for any domains $G \subset \mathbb{C}^{n}$ and $D \subset \mathbb{C}^{m}$, every holomorphic mapping $F: G \rightarrow D$ is a contraction with respect to $d_{G}$ and $d_{D}$, i.e.

$$
d_{D}(F(a), F(z)) \leq d_{G}(a, z), \quad a, z \in G .
$$

Let us recall some important holomorphically contractible families:

- Möbius pseudodistance:

$$
\begin{aligned}
c_{G}^{*}(a, z): & =\sup \left\{m_{E}(f(a), f(z)): f \in \mathcal{O}(G, E)\right\} \\
& =\sup \{|f(z)|: f \in \mathcal{O}(G, E), f(a)=0\},
\end{aligned}
$$

- higher order Möbius function:

$$
m_{G}^{(k)}(a, z):=\sup \left\{|f(z)|^{1 / k}: f \in \mathcal{O}(G, E), \operatorname{ord}_{a} f \geq k\right\}, \quad k \in \mathbb{N},
$$

where $\operatorname{ord}_{a} f$ denotes the order of zero of $f$ at $a$,

2000 Mathematics Subject Classification: 32F45, 32 U35.

Key words and phrases: generalized Green and Möbius functions, Lempert function, Coman function, holomorphically contractible family.

The first and third authors were supported by KBN grant no. 5 P03A 03321 and the Niedersächsisches Ministerium für Wissenschaft und Kultur, Az. 15.3-50 113(55) PL.

The second author was supported by KBN grant no. 2 P03A 01522. 
- pluricomplex Green function:

$$
\begin{aligned}
g_{G}(a, z):=\sup \{u(z): u: G \rightarrow & {[0,1), \log u \in \mathcal{P S H}(G), } \\
& \left.\exists_{C=C(u)>0} \forall_{w \in G}: u(w) \leq C\|w-a\|\right\},
\end{aligned}
$$

where $\mathcal{P} \mathcal{S H}(G)$ denotes the family of all functions plurisubharmonic on $G$,

- Lempert function:

$$
\begin{aligned}
\widetilde{k}_{G}^{*}(a, z): & =\inf \left\{m_{E}(\lambda, \mu): \exists_{\varphi \in \mathcal{O}(E, G)}: \varphi(\lambda)=a, \varphi(\mu)=z\right\} \\
& =\inf \left\{|\mu|: \exists_{\varphi \in \mathcal{O}(E, G)}: \varphi(0)=a, \varphi(\mu)=z\right\} .
\end{aligned}
$$

It is well known that

$$
c_{G}^{*}=m_{G}^{(1)} \leq m_{G}^{(k)} \leq g_{G} \leq \widetilde{k}_{G}^{*},
$$

and for any holomorphically contractible family $\left(d_{G}\right)_{G}$ we have

$$
c_{G}^{*} \leq d_{G} \leq \widetilde{k}_{G}^{*},
$$

i.e. the Möbius family is minimal and the Lempert family is maximal.

The Green function $g_{G}$ may be generalized as follows. Let $\boldsymbol{p}: G \rightarrow \mathbb{R}_{+}$ be an arbitrary function. Define

$$
\begin{aligned}
& g_{G}(\boldsymbol{p}, z):=\sup \{u(z): \quad u: G \rightarrow[0,1), \log u \in \mathcal{P} \mathcal{S H}(G), \\
&\left.\forall_{a \in G} \exists_{C=C(u, a)>0} \forall_{w \in G}: u(w) \leq C\|w-a\|^{\boldsymbol{p}(a)}\right\}, \quad z \in G\left(^{1}\right) ;
\end{aligned}
$$

obviously the above growth condition is trivially satisfied at all points $a \in G$ such that $\boldsymbol{p}(a)=0$. We have $g_{G}(\mathbf{0}, \cdot) \equiv 1$. The function $g_{G}(\boldsymbol{p}, \cdot)$ is called the generalized pluricomplex Green function with poles (weights) $\boldsymbol{p}$. Observe that if the set

$$
|\boldsymbol{p}|:=\{a \in G: \boldsymbol{p}(a)>0\}
$$

is not pluripolar, then $g_{G}(\boldsymbol{p}, \cdot) \equiv 0$.

In the case where $|\boldsymbol{p}|$ is finite, the function $g_{G}(\boldsymbol{p}, \cdot)$ was introduced by P. Lelong in [Lel 1989].

For $\boldsymbol{p}=\chi_{A}=$ the characteristic function of a set $A \subset G$, we put $g_{G}(A, \cdot):=g_{G}\left(\chi_{A}, \cdot\right)$. Obviously, $g_{G}(\{a\}, \cdot)=g_{G}(a, \cdot)$ for $a \in G$.

The generalized Green function was recently studied by many authors, e.g. [Car-Wie 2003], [Com 2000], [Edi 2002], [Edi-Zwo 1998], [Lár-Sig 1998].

Using the same idea, one can generalize the Möbius function. For

$$
\boldsymbol{p}: G \rightarrow \mathbb{Z}_{+}:=\{0,1,2, \ldots\}
$$

we put

$$
m_{G}(\boldsymbol{p}, z):=\sup \left\{|f(z)|: f \in \mathcal{O}(G, E), \operatorname{ord}_{a} f \geq \boldsymbol{p}(a), a \in G\right\}, \quad z \in G .
$$

The function $m_{G}(\boldsymbol{p}, \cdot)$ is called the generalized Möbius function with weights $\boldsymbol{p}$. Clearly $m_{G}(\mathbf{0}, \cdot) \equiv 1$. Observe that if the set $|\boldsymbol{p}|$ is not thin,

$\left({ }^{1}\right)$ Here $0^{0}:=1$. 
then $m_{G}(\boldsymbol{p}, \cdot) \equiv 0$. Similarly to the case of the generalized Green function we put $m_{G}(A, \cdot):=m_{G}\left(\chi_{A}, \cdot\right), A \subset G$. We get $m_{G}(\{a\}, \cdot)=c_{G}^{*}(a, \cdot), a \in G$. Moreover, if $|\boldsymbol{p}|=\{a\}$ and $\boldsymbol{p}(a)=k$, then $m_{G}(\boldsymbol{p}, \cdot)=\left[m_{G}^{(k)}(a, z)\right]^{k}$.

It is clear that $m_{G}(\boldsymbol{p}, \cdot) \leq g_{G}(\boldsymbol{p}, \cdot)$ (for any $\boldsymbol{p}: G \rightarrow \mathbb{Z}_{+}$). Some other properties of $g_{G}(\boldsymbol{p}, \cdot)$ and $m_{G}(\boldsymbol{p}, \cdot)$ will be presented in $\S 2$.

Definition 1.1. A family $\underline{d}=\left(d_{G}\right)_{G}$ of functions

$$
d_{G}: \mathbb{R}_{+}^{G} \times G \rightarrow \mathbb{R}_{+}
$$

is said to be a generalized holomorphically contractible family if the following three axioms are satisfied:

$$
\begin{aligned}
& \prod_{a \in E}\left[m_{E}(a, z)\right]^{\boldsymbol{p ( a )}} \leq d_{E}(\boldsymbol{p}, z) \leq \inf _{a \in E}\left[m_{E}(a, z)\right]^{\boldsymbol{p}(a)} \text { for every }(\boldsymbol{p}, z) \\
& \in \mathbb{R}_{+}^{E} \times E\left(^{2}\right),
\end{aligned}
$$

(H) for any $F \in \mathcal{O}(G, D)$ and $\boldsymbol{q}: D \rightarrow \mathbb{R}_{+}$we have

$$
d_{D}(\boldsymbol{q}, F(z)) \leq d_{G}(\boldsymbol{q} \circ F, z) \quad \text { for every } z \in G,
$$

(M) for any $\boldsymbol{p}, \boldsymbol{q}: G \rightarrow \mathbb{R}_{+}$, if $\boldsymbol{p} \leq \boldsymbol{q}$, then $d_{G}(\boldsymbol{q}, \cdot) \leq d_{G}(\boldsymbol{p}, \cdot)$.

If in the above definition one considers only integer-valued weights (as in the case of the generalized Möbius function), then we get the definition of a generalized holomorphically contractible family with integer-valued weights.

Put $d_{G}(A, \cdot):=d_{G}\left(\chi_{A}, \cdot\right), A \subset G, d_{G}(a, \cdot):=d_{G}(\{a\}, \cdot), a \in G$.

One can prove that the generalized Green and Möbius functions satisfy all the above axioms (cf. $\S 2$ ).

The main result of the paper is the following theorem.

THEOREM 1.2. In the category of generalized holomorphically contractible families there exists a minimal and a maximal object. They are given by the following formulae:

$$
\begin{aligned}
d_{G}^{\min }(\boldsymbol{p}, z) & :=\sup \left\{\prod_{\mu \in f(G)}\left[m_{E}(\mu, f(z))\right]^{\sup p\left(f^{-1}(\mu)\right)}: f \in \mathcal{O}(G, E)\right\} \\
& =\sup \left\{\prod_{\mu \in f(G)}|\mu|^{\sup p\left(f^{-1}(\mu)\right)}: f \in \mathcal{O}(G, E), f(z)=0\right\}, \\
d_{G}^{\max }(\boldsymbol{p}, z): & =\inf \left\{\left[\widetilde{k}_{G}^{*}(a, z)\right]^{p(a)}: a \in G\right\} \\
& =\inf \left\{|\mu|^{p(\varphi(\mu))}: \varphi \in \mathcal{O}(E, G), \varphi(0)=z, \mu \in E\right\} .
\end{aligned}
$$

Observe that if $|\boldsymbol{p}|=\{a\}$ and $\boldsymbol{p}(a)=k$, then $d_{G}^{\min }(\boldsymbol{p}, \cdot)=\left[c_{G}^{*}(a, \cdot)\right]^{k}$ and $d_{G}^{\max }(\boldsymbol{p}, \cdot)=\left[\widetilde{k}_{G}^{*}(a, \cdot)\right]^{k}$. Moreover, for $A \subset G$ we get

$\left({ }^{2}\right)$ We put $\prod_{a \in A} h(a):=\inf \left\{\prod_{a \in B} h(a): B \subset A, \# B<\infty\right\}$ for $h: A \rightarrow[0,1]$. 


$$
\begin{aligned}
d_{G}^{\min }(A, z) & =\sup \left\{\prod_{\mu \in f(A)} m_{E}(\mu, f(z)): f \in \mathcal{O}(G, E)\right\} \\
& =\sup \left\{\prod_{\mu \in f(A)}|\mu|: f \in \mathcal{O}(G, E), f(z)=0\right\}\left({ }^{3}\right), \\
d_{G}^{\max }(A, z) & =\inf \left\{\widetilde{k}_{G}^{*}(a, z): a \in A\right\} .
\end{aligned}
$$

The function $d_{G}^{\min }$ (resp. $d_{G}^{\max }$ ) may be considered as a generalization of the Möbius function $c_{G}^{*}$ (resp. Lempert function $\widetilde{k}_{G}^{*}$ ). The proof of Theorem 1.2 will be given in $\S 3$. Some properties of $d_{G}^{\min }$ and $d_{G}^{\max }$ will be presented in $\S 4$.

2. Basic properties of $g_{G}$ and $m_{G}$. Directly from the definitions we conclude that the systems $\left(g_{G}\right)_{G}$ and $\left(m_{G}\right)_{G}$ satisfy $(\mathrm{H})$ and $(\mathrm{M})$ and the following conditions (to simplify formulations we will write $d_{G}$ if a given property holds simultaneously for $m_{G}$ and $g_{G}$ ):

Property 2.1. We have

$$
d_{G}(\boldsymbol{p}, \cdot) d_{G}(\boldsymbol{q}, \cdot) \leq d_{G}(\boldsymbol{p}+\boldsymbol{q}, \cdot) \leq \min \left\{d_{G}(\boldsymbol{p}, \cdot), d_{G}(\boldsymbol{q}, \cdot)\right\} .
$$

In particular, $g_{G}(\boldsymbol{p}, \cdot) \leq \inf _{a \in G}\left[g_{G}(a, \cdot)\right]^{\boldsymbol{p}(a)} \leq d_{G}^{\max }(\boldsymbol{p}, \cdot)$.

Property 2.2. If the set $|\boldsymbol{p}|$ is finite, then

$$
\prod_{a \in G}\left[d_{G}(a, \cdot)\right]^{\boldsymbol{p}(a)} \leq d_{G}(\boldsymbol{p}, \cdot) .
$$

Property 2.3. We have

$$
\begin{aligned}
& g_{G}(\boldsymbol{p}, z)=\sup \{u(z): \quad u: G \rightarrow[0,1), \log u \in \mathcal{P} \mathcal{S H}(G), \\
&\left.u(\cdot) \leq \inf _{a \in G}\left[g_{G}(a, \cdot)\right]^{\boldsymbol{p}(a)}\right\}, \quad z \in G .
\end{aligned}
$$

Property 2.4. $m_{G}(\boldsymbol{p}, \cdot) \in \mathcal{C}(G)$.

Proof. The family $\left\{f \in \mathcal{O}(G, E): \operatorname{ord}_{a} f \geq \boldsymbol{p}(a), a \in G\right\}$ is equicontinuous.

PROPERTy 2.5. If $\boldsymbol{p} \not \equiv 0$, then for any $z_{0} \in G$ there exists an extremal function for $m_{G}\left(\boldsymbol{p}, z_{0}\right)$, i.e. a function $f_{z_{0}} \in \mathcal{O}(G, E)$ with $\operatorname{ord}_{a} f_{z_{0}} \geq \boldsymbol{p}(a)$, $a \in G$, and $m_{G}\left(\boldsymbol{p}, z_{0}\right)=\left|f_{z_{0}}\left(z_{0}\right)\right|$.

Property 2.6. $\log d_{G}(\boldsymbol{p}, \cdot) \in \mathcal{P} \mathcal{S H}(G)$.

Proof. Argue as in the one-pole case (cf. [Jar-Pfl 1993, §§2.5, 4.2]).

PROPERTy 2.7. If $G_{k} \nearrow G$ and $\boldsymbol{p}_{k} \nearrow \boldsymbol{p}$, then $d_{G_{k}}\left(\boldsymbol{p}_{k}, z\right) \searrow d_{G}(\boldsymbol{p}, z)$ for $z \in G$.

$\left({ }^{3}\right)$ In fact, $d_{G}^{\min }(A, \cdot)=m_{G}(A, \cdot)($ cf. Corollary 3.1(c)). 
Proof. It is clear that the sequence is monotone and the limit function $u$ satisfies $u \geq d_{G}(\boldsymbol{p}, \cdot)$.

In the case of the generalized Green function, using 2.6, we have $u \in \mathcal{P S H}(G)$. By 2.3 it remains to observe that $u(z) \leq \inf _{a \in G}\left[g_{G}(a, z)\right]^{p(a)}$ for $z \in G$ (because $g_{G_{k}}(a, z) \searrow g_{G}(a, z)$ for every $\left.(a, z) \in G \times G\right)$.

The case of the generalized Möbius function is simpler and it follows from 2.5 and a Montel argument.

Property 2.8. $g_{G}(\boldsymbol{p}, \cdot)=\inf \left\{g_{G}(\boldsymbol{q}, \cdot): \boldsymbol{q} \leq \boldsymbol{p}, \#|\boldsymbol{q}|<\infty\right\}$.

Proof. Let $u:=\inf \left\{g_{G}(\boldsymbol{q}, \cdot): \boldsymbol{q} \leq \boldsymbol{p}, \#|\boldsymbol{q}|<\infty\right\}$. Obviously $g_{G}(\boldsymbol{p}, \cdot) \leq u$. By 2.3 , to prove the opposite inequality we only need to show that $\log u$ is plurisubharmonic. Observe that

$$
g_{G}\left(\max \left\{\boldsymbol{q}_{1}, \ldots, \boldsymbol{q}_{N}\right\}, \cdot\right) \leq \min \left\{g_{G}\left(\boldsymbol{q}_{1}, \cdot\right), \ldots, g_{G}\left(\boldsymbol{q}_{N}, \cdot\right)\right\} .
$$

We finish the proof by applying the following general result.

Lemma 2.9. Let $\left(v_{i}\right)_{i \in A} \subset \mathcal{P S H}(\Omega)\left(\Omega \subset \mathbb{C}^{n}\right)$ be such that for any $i_{1}, \ldots, i_{N} \in A$ there exists an $i_{0} \in A$ such that $v_{i_{0}} \leq \min \left\{v_{i_{1}}, \ldots, v_{i_{N}}\right\}$. Then $v:=\inf _{i \in A} v_{i} \in \mathcal{P} \mathcal{S H}(\Omega)$.

Proof. It suffices to consider the case $n=1$. Take a disc $\Delta_{a}(r) \Subset \Omega$, $\varepsilon>0$, and a continuous function $w \in \mathcal{C}\left(\partial \Delta_{a}(r)\right)$ such that $w \geq v$ on $\partial \Delta_{a}(r)$. We want to show that $v(a) \leq(2 \pi)^{-1} \int_{0}^{2 \pi} w\left(a+r e^{i \theta}\right) d \theta+\varepsilon$. For any point $b \in \partial \Delta_{a}(r)$ there exists an $i=i(b) \in A$ such that $v_{i}(b)<w(b)+\varepsilon$. Hence there exists an open arc $I=I(b) \subset \partial \Delta_{a}(r)$ with $b \in I$ such that $v_{i}(\lambda)<$ $w(\lambda)+\varepsilon$ for $\lambda \in I$. By a compactness argument, we find $b_{1}, \ldots, b_{N} \in \partial \Delta_{a}(r)$ such that $\partial \Delta_{a}(r)=\bigcup_{j=1}^{N} I\left(b_{j}\right)$. By assumption, there exists an $i_{0} \in A$ such that $v_{i_{0}} \leq \min \left\{v_{i\left(b_{1}\right)}, \ldots, v_{i\left(b_{N}\right)}\right\}$. Then

$$
\begin{aligned}
v(a) \leq v_{i_{0}}(a) & \leq \frac{1}{2 \pi} \int_{0}^{2 \pi} v_{i_{0}}\left(a+r e^{i \theta}\right) d \theta \\
& \leq \frac{1}{2 \pi} \int_{0}^{2 \pi} w\left(a+r e^{i \theta}\right) d \theta+\varepsilon .
\end{aligned}
$$

Property 2.10. We have

$$
\prod_{a \in G}\left[g_{G}(a, \cdot)\right]^{p(a)} \leq g_{G}(\boldsymbol{p}, \cdot) .
$$

Proof. Use 2.2 and 2.8 .

Property 2.11. If $G \subset \mathbb{C}$, then

$$
g_{G}(\boldsymbol{p}, z)=\prod_{a \in G}\left[g_{G}(a, z)\right]^{p(a)}, \quad z \in G .
$$

In particular, $g_{E}(\boldsymbol{p}, z)=\prod_{a \in E}\left[m_{E}(a, z)\right]^{p(a)}$ for $z \in E$. 
Proof. By 2.8 we may assume that the set $|\boldsymbol{p}|$ is finite, and by 2.7, that $G \Subset \mathbb{C}$ is regular with respect to the Dirichlet problem. Let $u:=$ $\prod_{a \in|\boldsymbol{p}|}\left[g_{G}(a, \cdot)\right]^{\boldsymbol{p}(a)}$. Then $\log u$ is subharmonic on $G$ and harmonic on $G \backslash|\boldsymbol{p}|$. The function $v:=\log g_{G}(\boldsymbol{p}, \cdot)-\log u$ is locally bounded from above in $G$ and $\limsup _{z \rightarrow \zeta} v(z) \leq 0$ for $\zeta \in \partial G$. Consequently, $v$ extends to a subharmonic function on $G$, and by the maximum principle, $v \leq 0$ on $G$, i.e. $g_{G}(\boldsymbol{p}, \cdot) \leq u$ on $G$. The opposite inequality follows from 2.10 .

Property 2.12. For any $\boldsymbol{p}: G \rightarrow \mathbb{Z}_{+}$,

$$
m_{G}(\boldsymbol{p}, \cdot)=\inf \left\{m_{G}(\boldsymbol{q}, \cdot): \boldsymbol{q}: G \rightarrow \mathbb{Z}_{+}, \boldsymbol{q} \leq \boldsymbol{p}, \#|\boldsymbol{q}|<\infty\right\} .
$$

In particular, for any $\boldsymbol{p}: E \rightarrow \mathbb{Z}_{+}$,

$$
m_{E}(\boldsymbol{p}, z)=g_{E}(\boldsymbol{p}, z)=\prod_{a \in E}\left[m_{E}(a, z)\right]^{\boldsymbol{p}(a)}, \quad z \in E .
$$

Proof. The case where $|\boldsymbol{p}|$ is finite is trivial; the case where it is countable follows from 2.7. In the general case let $A_{k}:=\{a \in G: \boldsymbol{p}(a)=k\}$ and let $B_{k}$ be a countable (or finite) dense subset of $A_{k}$ for $k \in \mathbb{Z}_{+}$. Put $B:=\bigcup_{k=0}^{\infty} B_{k}$ and $\boldsymbol{p}^{\prime}:=\boldsymbol{p} \cdot \chi_{B}$. Then $\boldsymbol{p}^{\prime} \leq \boldsymbol{p}$, the set $\left|\boldsymbol{p}^{\prime}\right|$ is countable, and $m_{G}(\boldsymbol{p}, \cdot) \equiv$ $m_{G}\left(\boldsymbol{p}^{\prime}, \cdot\right)$. Consequently, the result reduces to the countable case.

Proposition 2.13 ([Edi-Zwo 1998], [Lár-Sig 1998]). Let $G, D \subset \mathbb{C}^{n}$ be domains and let $F: G \rightarrow D$ be a proper holomorphic mapping. Let $\boldsymbol{q}: D \rightarrow \mathbb{R}_{+}$. Assume that $\operatorname{det} F^{\prime}(a) \neq 0$ for any $a \in G$ such that $\boldsymbol{q}(F(a))>0$. Then

$$
g_{D}(\boldsymbol{q}, F(z))=g_{G}(\boldsymbol{q} \circ F, z), \quad z \in G .
$$

In particular, if $B \subset D$ is such that $\operatorname{det} F^{\prime}(a) \neq 0$ for any $a \in F^{-1}(B)$, then

$$
g_{D}(B, F(z))=g_{G}\left(F^{-1}(B), z\right), \quad z \in G .
$$

Corollary 2.14. Let $A_{1}, \ldots, A_{n} \subset E$ be finite sets. Put

$$
\begin{gathered}
F_{j}(\lambda):=\prod_{a \in A_{j}} \frac{\lambda-a}{1-\bar{a} \lambda}, \quad \lambda \in E, j=1, \ldots, n, \\
F(z):=\left(F_{1}\left(z_{1}\right), \ldots, F_{n}\left(z_{n}\right)\right), \quad z=\left(z_{1}, \ldots, z_{n}\right) \in E^{n} .
\end{gathered}
$$

Then

$$
\begin{aligned}
m_{E^{n}}\left(A_{1} \times \ldots \times A_{n}, z\right) & \leq g_{E^{n}}\left(A_{1} \times \ldots \times A_{n}, z\right) \\
& =g_{E^{n}}(0, F(z))=\max \left\{\left|F_{j}\left(z_{j}\right)\right|: j=1, \ldots, n\right\} \\
& =\max \left\{m_{E}\left(A_{1}, z_{1}\right), \ldots, m_{E}\left(A_{n}, z_{n}\right)\right\} \\
& \leq m_{E^{n}}\left(A_{1} \times \ldots \times A_{n}, z\right), \quad z=\left(z_{1}, \ldots, z_{n}\right) \in E^{n} .
\end{aligned}
$$


Proposition 2.15 ([Car-Wie 2003]). Let $\boldsymbol{p}: E^{n} \rightarrow \mathbb{R}_{+}$be such that $|\boldsymbol{p}|=\left\{a_{1}, \ldots, a_{N}\right\} \subset E \times\{0\}^{n-1}$. Put $k_{j}:=\boldsymbol{p}\left(a_{j}\right), j=1, \ldots, N$, and assume that $k_{1} \geq \ldots \geq k_{N}$. Then

$$
g_{E^{n}}(\boldsymbol{p}, z)=\prod_{j=1}^{N} u_{j}^{k_{j}-k_{j+1}}(z), \quad z \in E^{n},
$$

where $k_{N+1}:=0$ and

$$
\begin{aligned}
u_{j}(z) & :=\max \left\{m_{E}\left(a_{1,1}, z_{1}\right) \ldots m_{E}\left(a_{j, 1}, z_{1}\right),\left|z_{2}\right|, \ldots,\left|z_{n}\right|\right\} \\
& =\max \left\{m_{E}\left(\left\{a_{1,1}, \ldots, a_{j, 1}\right\}, z_{1}\right),\left|z_{2}\right|, \ldots,\left|z_{n}\right|\right\} \\
& =g_{E^{n}}\left(\left\{a_{1}, \ldots, a_{j}\right\}, z\right), \quad j=1, \ldots, N .
\end{aligned}
$$

If $k_{1}, \ldots, k_{N} \in \mathbb{N}$, then $m_{E^{n}}(\boldsymbol{p}, \cdot)=g_{E^{n}}(\boldsymbol{p}, \cdot)$.

Observe that if $k_{1}=\ldots=k_{N}=1$, then the above formula coincides with that from Corollary 2.14.

Notice that even for the simplest case not covered by Proposition 2.15: $n=N=2, a_{1}=(0,0), a_{2} \in\left(E_{*}\right)^{2}, k_{1}=k_{2}=1$, an effective formula for $g_{E^{n}}(\boldsymbol{p}, \cdot)$ is not known.

Recall that by the Lempert theorem (cf. [Jar-Pfl 1993, Ch. 8]), if $G \subset \mathbb{C}^{n}$ is convex, then $c_{G}^{*}=\widetilde{k}_{G}^{*}$, and consequently, by $(*)$, all holomorphically contractible families coincide on $G$. The following example shows that this is not true in the category of generalized holomorphically contractible families.

Example 2.16 (due to W. Zwonek). Let $D:=\left\{(z, w) \in \mathbb{C}^{2}:|z|+|w|<1\right\}$, $A_{t}:=\{(t, \sqrt{t}),(t,-\sqrt{t})\}, 0<t \ll 1$. Then

$$
m_{D}\left(A_{t},(0,0)\right)<g_{D}\left(A_{t},(0,0)\right)<d_{D}^{\max }\left(A_{t},(0,0)\right)
$$

for small $t$.

Indeed, let $G:=\left\{(z, w) \in \mathbb{C}^{2}:|z|+\sqrt{|w|}<1\right\}$ and let $F: D \rightarrow G$, $F(z, w):=\left(z, w^{2}\right)$. Note that $F$ is proper and locally biholomorphic in a neighborhood of $A_{t}$. Moreover, $A_{t}=F^{-1}(t, t)$.

Using Proposition 2.13, we conclude that $g_{D}\left(A_{t},(0,0)\right)=g_{G}((t, t),(0,0))$.

Observe that $m_{D}\left(A_{t},(0,0)\right)=m_{G}((t, t),(0,0))$. In fact, the inequality " $\geq$ " follows from $(\mathrm{H})$ (applied to $F$ ). The opposite inequality may be proved as follows. Let $f \in \mathcal{O}(D, E)$ be such that $\left.f\right|_{A_{t}}=0$. Define

$$
\widetilde{f}(z, w):=\frac{1}{2}(f(z, \sqrt{w})+f(z,-\sqrt{w})), \quad(z, w) \in G .
$$

Note that $\tilde{f}$ is well defined, $|\widetilde{f}|<1, \widetilde{f}(t, t)=0, \widetilde{f}$ is continuous, and $\widetilde{f}$ 
is holomorphic on $D \cap\{w \neq 0\}$. In particular, $\widetilde{f}$ is holomorphic on $D$. Consequently, $|f(0,0)|=|\widetilde{f}(0,0)| \leq m_{G}((t, t),(0,0))$. Then

Suppose that $m_{D}\left(A_{t_{k}},(0,0)\right)=g_{D}\left(A_{t_{k}},(0,0)\right)$ for a sequence $t_{k} \searrow 0$.

$$
\begin{aligned}
g_{G}\left(\left(t_{k}, t_{k}\right),(0,0)\right) & =g_{D}\left(A_{t_{k}},(0,0)\right)=m_{D}\left(A_{t_{k}},(0,0)\right) \\
& =m_{G}\left(\left(t_{k}, t_{k}\right),(0,0)\right) \leq g_{G}\left(\left(t_{k}, t_{k}\right),(0,0)\right), \quad k=1,2, \ldots
\end{aligned}
$$

Thus $m_{G}\left(\left(t_{k}, t_{k}\right),(0,0)\right)=g_{G}\left(\left(t_{k}, t_{k}\right),(0,0)\right), k=1,2, \ldots$

Consequently, using [Jar-Pfl 1993, §2.5], and [Zwo 2000a, Corollary 4.4] (or [Zwo 2000b, Corollary 4.2.3]), we conclude that

$$
\gamma_{G}((0,0) ;(1,1))=A_{G}((0,0) ;(1,1)),
$$

where $\gamma_{G}$ (resp. $A_{G}$ ) denotes the Carathéodory-Reiffen (resp. Azukawa) metric of $G$ (cf. [Jar-Pfl 1993, $\S \S 2.1,4.2]$ ). Hence, by Propositions 4.2.7 and 2.2.1(d) from [Jar-Pfl 1993], using the fact that $D$ is the convex envelope of $G$, we get

$$
2=h_{D}(1,1)=\gamma_{G}((0,0) ;(1,1))=A_{G}((0,0) ;(1,1))=h_{G}(1,1)=\frac{2}{3-\sqrt{5}},
$$

where $h_{D}\left(\right.$ resp. $\left.h_{G}\right)$ denotes the Minkowski function for $D$ (resp. $G$ ); contradiction.

To prove the inequality $g_{D}\left(A_{t},(0,0)\right)<d_{D}^{\max }\left(A_{t},(0,0)\right)$, we may argue as follows. We already know that

$$
\begin{aligned}
g_{D}\left(A_{t},(0,0)\right) & =g_{G}((t, t),(0,0)) \\
& \approx g_{G}((0,0),(t, t))=h_{G}(t, t)=\frac{2 t}{3-\sqrt{5}}, \quad t \approx 0 .
\end{aligned}
$$

On the other hand,

$$
\begin{aligned}
d_{D}^{\max }\left(A_{t},(0,0)\right) & =\min \left\{\widetilde{k}_{D}^{*}((t,-\sqrt{t}),(0,0)), \widetilde{k}_{D}^{*}((t, \sqrt{t}),(0,0))\right\} \\
& =\min \left\{h_{D}(t,-\sqrt{t}), h_{D}(t, \sqrt{t})\right\}=t+\sqrt{t} .
\end{aligned}
$$

It remains to observe that $2 t /(3-\sqrt{5})<t+\sqrt{t}$ for small $t>0$.

Let $\delta_{D}\left(A_{t}, \cdot\right)$ denote the Coman function for $D$ with poles at $A_{t}$, i.e.

$$
\begin{aligned}
& \delta_{D}\left(A_{t},(z, w)\right)=\inf \left\{\left|\mu_{1} \mu_{2}\right|: \exists_{\varphi \in \mathcal{O}(E, D)}:\right. \\
& \left.\quad \varphi(0)=(z, w), \varphi\left(\mu_{1}\right)=(t, \sqrt{t}), \varphi\left(\mu_{2}\right)=(t,-\sqrt{t})\right\}, \quad(z, w) \in D
\end{aligned}
$$

(cf. [Com 2000]). It is known that $g_{D}\left(A_{t}, \cdot\right) \leq \delta_{D}\left(A_{t}, \cdot\right)$. Taking $\varphi(\lambda):=$ $\left(\lambda^{2} / 4, \lambda / 2\right)$, we easily see that $\delta_{D}\left(A_{t},(0,0)\right) \leq 4 t<t+\sqrt{t}=d_{D}^{\max }\left(A_{t},(0,0)\right)$, $0<t \ll 1$. We do not know whether $g_{D}\left(A_{t},(0,0)\right)<\delta_{D}\left(A_{t},(0,0)\right)$ for small $t>0$. 


\section{Proof of Theorem 1.2}

SteP 1. If $\left(d_{G}\right)_{G}$ satisfies $(\mathrm{H})$ and

$\left(\mathrm{E}^{+}\right) \quad d_{E}(\boldsymbol{p}, \lambda) \leq d_{E}^{\max }(\boldsymbol{p}, \lambda)=\inf \left\{\left[m_{E}(\mu, \lambda)\right]^{\boldsymbol{p}(\mu)}: \mu \in E\right\}, \quad(\boldsymbol{p}, \lambda) \in$ $\mathbb{R}_{+}^{E} \times E$, then $d_{G} \leq d_{G}^{\max }$ for any $G$. The same remains true in the category of contractible families with integer-valued weights.

Proof. We have

$$
\begin{aligned}
d_{G}(\boldsymbol{p}, z) & \stackrel{(\mathrm{H})}{\leq} \inf \left\{d_{E}(\boldsymbol{p} \circ \varphi, 0): \varphi \in \mathcal{O}(E, G), \varphi(0)=z\right\} \\
& \stackrel{\left(\mathrm{E}^{+}\right)}{\leq} \inf \left\{|\mu|^{p(\varphi(\mu))}: \varphi \in \mathcal{O}(E, G), \varphi(0)=z, \mu \in E\right\} \\
& =d_{G}^{\max }(\boldsymbol{p}, z), \quad(\boldsymbol{p}, z) \in \mathbb{R}_{+}^{G} \times G .
\end{aligned}
$$

SteP 2. The system $\left(d_{G}^{\max }\right)_{G}$ satisfies $(\mathrm{E}),(\mathrm{H})$, and $(\mathrm{M})$.

Proof. (E) and (M) are obvious. To prove (H) let $F: G \rightarrow D$ be holomorphic and let $\boldsymbol{q}: D \rightarrow \mathbb{R}_{+}$. Then

$$
\begin{aligned}
d_{D}^{\max }(\boldsymbol{q}, F(z)) & =\inf \left\{\left[\widetilde{k}_{D}^{*}(b, F(z))\right]^{\boldsymbol{q}(b)}: b \in D\right\} \\
& \leq \inf \left\{\left[\widetilde{k}_{D}^{*}(F(a), F(z))\right]^{\boldsymbol{q}(F(a))}: a \in G\right\} \\
& \leq \inf \left\{\left[\widetilde{k}_{G}^{*}(a, z)\right]^{\boldsymbol{q}(F(a))}: a \in G\right\}=d_{G}^{\max }(\boldsymbol{q} \circ F, z), \quad z \in G .
\end{aligned}
$$

STEP 3. If $\left(d_{G}\right)_{G}$ satisfies $(\mathrm{H}),(\mathrm{M})$, and

$$
\prod_{\mu \in E}\left[m_{E}(\mu, \lambda)\right]^{p(\mu)} \leq d_{E}(\boldsymbol{p}, \lambda), \quad(\boldsymbol{p}, \lambda) \in \mathbb{R}_{+}^{E} \times E,
$$

then $d_{G}^{\min } \leq d_{G}$ for any $G$. The same remains true in the category of contractible families with integer-valued weights.

Proof. Indeed,

$d_{G}(\boldsymbol{p}, z)$

$$
\begin{aligned}
& \stackrel{(\mathrm{M})}{\geq} \sup \left\{d_{G}(\boldsymbol{q} \circ f, z): f \in \mathcal{O}(G, E), \boldsymbol{q}: E \rightarrow \mathbb{R}_{+}, f(z)=0, \boldsymbol{p} \leq \boldsymbol{q} \circ f\right\} \\
& \stackrel{(\mathrm{H})}{\geq} \sup \left\{d_{E}(\boldsymbol{q}, 0): f \in \mathcal{O}(G, E), \boldsymbol{q}: E \rightarrow \mathbb{R}_{+}, f(z)=0, \boldsymbol{p} \leq \boldsymbol{q} \circ f\right\} \\
& \stackrel{\left(\mathrm{E}^{-}\right)}{\geq} \sup \left\{\prod_{\mu \in E}|\mu|^{\boldsymbol{q}(\mu)}: f \in \mathcal{O}(G, E), \boldsymbol{q}: E \rightarrow \mathbb{R}_{+}, f(z)=0, \boldsymbol{p} \leq \boldsymbol{q} \circ f\right\} \\
& \geq \sup \left\{\prod_{\mu \in f(G)}|\mu|^{\sup \boldsymbol{p}\left(f^{-1}(\mu)\right)}: f \in \mathcal{O}(G, E), f(z)=0\right\} \\
& =d_{G}^{\min }(\boldsymbol{p}, z), \quad(\boldsymbol{p}, z) \in \mathbb{R}_{+}^{G} \times G .
\end{aligned}
$$

SteP 4. The system $\left(d_{G}^{\mathrm{min}}\right)_{G}$ satisfies $(\mathrm{E}),(\mathrm{H})$, and $(\mathrm{M})$. 
Proof. (E) and (M) are elementary. To prove (H) let $F: G \rightarrow D$ be holomorphic and let $\boldsymbol{q}: D \rightarrow \mathbb{R}_{+}$. Then

$$
\begin{aligned}
& d_{D}^{\min }(\boldsymbol{q}, F(z))=\sup \left\{\prod_{\mu \in g(D)}\left[m_{E}(\mu, g(F(z))]^{\sup \boldsymbol{q}\left(g^{-1}(\mu)\right)}: g \in \mathcal{O}(D, E)\right\}\right. \\
& \stackrel{f=g \circ F}{\leq} \sup \left\{\prod_{\mu \in f(G)}\left[m_{E}(\mu, f(z))\right]^{\sup (\boldsymbol{q} \circ F)\left(f^{-1}(\mu)\right)}: f \in \mathcal{O}(G, E)\right\} \\
&=d_{G}^{\min }(\boldsymbol{q} \circ F, z), \quad z \in G .
\end{aligned}
$$

Corollary 3.1. (a) $d_{G}^{\min } \leq g_{G} \leq d_{G}^{\max }$ and $d_{G}^{\min } \leq m_{G} \leq g_{G} \leq d_{G}^{\max }$ (for integer-valued weights).

(b) $d_{E}^{\min }(\boldsymbol{p}, \lambda)=g_{E}(\boldsymbol{p}, \lambda)=\prod_{\mu \in E}\left[m_{E}(\mu, \lambda)\right]^{p(\mu)}$ for $(\boldsymbol{p}, \lambda) \in \mathbb{R}_{+}^{E} \times E$.

(c) $d_{G}^{\min }(A, \cdot)=m_{G}(A, \cdot)$ for any $A \subset G$.

Proof. (a) follows from Theorem 1.2.

(b) Using (a) and 2.11 we get

$$
\prod_{\mu \in E}\left[m_{E}(\mu, \lambda)\right]^{p(\mu)} \leq d_{E}^{\min }(\boldsymbol{p}, \lambda) \leq g_{G}(\boldsymbol{p}, \lambda)=\prod_{\mu \in E}\left[m_{E}(\mu, \lambda)\right]^{\boldsymbol{p}(\mu)} .
$$

(c) Let $A \subset G$. Then

$$
\begin{aligned}
m_{G}(A, z) & \geq d_{G}^{\min }(A, z) \\
& \geq \sup \left\{\prod_{\mu \in f(A)} m_{E}(\mu, f(z)): f \in \mathcal{O}(G, E),\left.f\right|_{A}=0\right\} \\
& =m_{G}(A, z), \quad z \in G .
\end{aligned}
$$

EXAMPLE 3.2. Let $G:=E^{2}, a_{-}:=\left(-\frac{1}{2}, 0\right), a_{+}:=\left(\frac{1}{2}, 0\right), b:=\left(0, \frac{1}{3}\right)$, $|\boldsymbol{p}|=\left\{a_{-}, a_{+}\right\}, \boldsymbol{p}\left(a_{-}\right)=2, \boldsymbol{p}\left(a_{+}\right)=1$. Then $d_{E^{2}}^{\min }(\boldsymbol{p}, b)<m_{E^{2}}(\boldsymbol{p}, b)$ (cf. Corollary 3.1(c)).

Indeed, by Proposition 2.15,

$$
m_{E^{2}}(\boldsymbol{p}, b)=u_{1}(b) u_{2}(b)=\max \left\{\frac{1}{2}, \frac{1}{3}\right\} \max \left\{\frac{1}{2} \cdot \frac{1}{2}, \frac{1}{3}\right\}=\frac{1}{2} \cdot \frac{1}{3}=\frac{1}{6} .
$$

On the other hand,

$$
\begin{aligned}
& d_{E^{2}}^{\min }(\boldsymbol{p}, b) \\
& =\max \left\{\sup \left\{\left|f\left(a_{-}\right)\right|^{2}\left|f\left(a_{+}\right)\right|: f \in \mathcal{O}\left(E^{2}, E\right), f(b)=0, f\left(a_{-}\right) \neq f\left(a_{+}\right)\right\}\right. \\
& \left.\quad \quad \sup \left\{|f(b)|^{2}: f \in \mathcal{O}\left(E^{2}, E\right), f\left(a_{-}\right)=f\left(a_{+}\right)=0\right\}\right\} \\
& \leq \max \left\{\left[m_{E^{2}}\left(a_{-}, b\right)\right]^{2} m_{E^{2}}\left(a_{+}, b\right),\left[m_{E^{2}}\left(\left\{a_{-}, a_{+}\right\}, b\right)\right]^{2}\right\} \\
& =\max \left\{\left[\max \left\{\frac{1}{2}, \frac{1}{3}\right\}\right]^{2} \max \left\{\frac{1}{2}, \frac{1}{3}\right\},\left[m_{E^{2}}\left(\left\{-\frac{1}{2}, \frac{1}{2}\right\} \times\{0\}, b\right)\right]^{2}\right\} \\
& =\max \left\{\frac{1}{8},\left[\max \left\{\frac{1}{2} \cdot \frac{1}{2}, \frac{1}{3}\right\}\right]^{2}\right\}=\frac{1}{8} .
\end{aligned}
$$




\section{Basic properties of $d_{G}^{\min }$ and $d_{G}^{\max }$}

Property 4.1. If $D \subset \mathbb{C}^{m}$ is a Liouville domain, then

$$
d_{G \times D}^{\min }(\boldsymbol{p},(z, w))=d_{G}^{\min }\left(\boldsymbol{p}^{\prime}, z\right), \quad(z, w) \in G \times D,
$$

where $\boldsymbol{p}^{\prime}(z):=\sup \{\boldsymbol{p}(z, w): w \in D\}, z \in G$, and $d_{G}^{\min }\left(\boldsymbol{p}^{\prime}, \cdot\right):=0$ if there exists a $z_{0} \in G$ with $\boldsymbol{p}^{\prime}\left(z_{0}\right)=\infty$.

Property 4.2. (a) The functions $d_{G}^{\min }(\boldsymbol{p}, \cdot)$ and $d_{G}^{\max }(\boldsymbol{p}, \cdot)$ are upper semicontinuous.

(b) If $\boldsymbol{p}: G \rightarrow \mathbb{Z}_{+}$, then $d_{G}^{\min }(\boldsymbol{p}, \cdot) \in \mathcal{C}(G)$ (cf. 2.4).

Proof. (a) The case of $d_{G}^{\max }(\boldsymbol{p}, \cdot)$ is obvious. To prove the upper semicontinuity of $d_{G}^{\min }(\boldsymbol{p}, \cdot)$, fix a $z_{0} \in G$ and suppose that

$$
d_{G}^{\min }\left(\boldsymbol{p}, z_{k}\right) \rightarrow \alpha>\beta>d_{G}^{\min }\left(\boldsymbol{p}, z_{0}\right)
$$

for some sequence $z_{k} \rightarrow z_{0}$. Let $f_{k} \in \mathcal{O}(G, E)$ be such that $f_{k}\left(z_{k}\right)=0$ and $\prod_{\mu \in f_{k}(G)}|\mu|^{\sup p\left(f_{k}^{-1}(\mu)\right)} \rightarrow \alpha$. By a Montel argument we may assume that $f_{k} \rightarrow f_{0}$ locally uniformly in $G$ with $f_{0} \in \mathcal{O}(G, E), f_{0}\left(z_{0}\right)=0$. Since $\prod_{\mu \in f_{0}(G)}|\mu|^{\sup p\left(f_{0}^{-1}(\mu)\right)}<\beta$, we can find a finite set $A \subset G$ such that $\left.f_{0}\right|_{A}$ is injective and $\prod_{a \in A}\left|f_{0}(a)\right|^{p(a)}<\beta$. Consequently, $\prod_{a \in A}\left|f_{k}(a)\right|^{p(a)}<\beta$ and $\left.f_{k}\right|_{A}$ is injective for $k \gg 1$. Finally, $\prod_{\mu \in f_{k}(G)}|\mu|^{\sup p\left(f_{k}^{-1}(\mu)\right)}<\beta$ for $k \gg 1$; contradiction.

(b) In view of (a), it suffices to prove that for every $f \in \mathcal{O}(G, E)$ the function $u_{f}(z):=\prod_{\mu \in f(G)}\left[m_{E}(\mu, f(z))\right]^{\sup p\left(f^{-1}(\mu)\right)}, z \in G$, is continuous on $G$. Observe that $u_{f}(z)=\inf _{M} \prod_{\mu \in M}\left[m_{E}(\mu, f(z))\right]^{k_{f}(\mu)}$, where $M$ runs over all finite sets $M \subset f(|\boldsymbol{p}|)$ such that $k_{f}(\mu):=\sup \boldsymbol{p}\left(f^{-1}(\mu)\right)<\infty$, $\mu \in M$. Thus $u_{f}=\inf _{M}\left|h_{M}\right|$, where $h_{M} \in \mathcal{O}(G, E)$. Consequently, since the family $\left(h_{M}\right)_{M}$ is equicontinuous, the function $u_{f}$ is continuous on $G$.

EXAMPLE 4.3. Let $\boldsymbol{p}: E \times \mathbb{C} \rightarrow \mathbb{R}_{+}$be defined by $\boldsymbol{p}(1 / k, k):=1 / k^{2}$ for $k=2,3, \ldots$, and $\boldsymbol{p}(z, w):=0$ otherwise. Notice that $|\boldsymbol{p}|$ is discrete. Then by 4.1 and Corollary 3.1(b),

$$
d_{E \times \mathbb{C}}^{\min }(\boldsymbol{p},(z, w))=d_{E}^{\min }\left(\boldsymbol{p}^{\prime}, z\right)=\prod_{k=2}^{\infty}\left[m_{E}(1 / k, z)\right]^{1 / k^{2}}, \quad(z, w) \in E \times \mathbb{C} .
$$

In particular, $d_{E \times \mathbb{C}}^{\min }(\boldsymbol{p}, \cdot)$ is discontinuous at $(0, w) \in E \times \mathbb{C} \backslash|\boldsymbol{p}|$.

Property 4.4 (cf. 2.5). If $\#|\boldsymbol{p}|<\infty$, then for any $z_{0} \in G$ there exists an extremal function for $d_{G}^{\min }\left(\boldsymbol{p}, z_{0}\right)$, i.e. a function $f_{z_{0}} \in \mathcal{O}(G, E)$ with $f_{z_{0}}\left(z_{0}\right)=0$ and

$$
\prod_{\mu \in f_{z_{0}}(G)}|\mu|^{\sup p\left(f_{z_{0}}^{-1}(\mu)\right)}=d_{G}^{\min }\left(\boldsymbol{p}, z_{0}\right)
$$


Proof. Fix a $z_{0} \in G$ and let $f_{k} \in \mathcal{O}(G, E)$ with $f_{k}\left(z_{0}\right)=0$ be such that

$$
\alpha_{k}:=\prod_{\mu \in f_{k}(G)}|\mu|^{\sup p\left(f_{k}^{-1}(\mu)\right)} \rightarrow \alpha:=d_{G}^{\min }\left(\boldsymbol{p}, z_{0}\right) .
$$

Let $A_{k} \subset|\boldsymbol{p}|$ be such that $\left.f_{k}\right|_{A_{k}}$ is injective, $f_{k}\left(A_{k}\right)=f_{k}(|\boldsymbol{p}|)$, and $\boldsymbol{p}(a)=$ $\sup \boldsymbol{p}\left(f_{k}^{-1}\left(f_{k}(a)\right)\right)$ for $a \in A_{k}$. Thus $\alpha_{k}=\prod_{a \in A_{k}}\left|f_{k}(a)\right|^{\boldsymbol{p}(a)}$. We may assume that $A_{k}=B$ is independent of $k$ and for any $a \in B$ the fiber $B_{a}:=f_{k}^{-1}\left(f_{k}(a)\right) \cap|\boldsymbol{p}|$ is also independent of $k$. Moreover, we may assume that $f_{k} \rightarrow f_{0}$ locally uniformly in $G$. Then $f_{0} \in \mathcal{O}(G, E), f_{0}\left(z_{0}\right)=0$, and $\prod_{a \in B}\left|f_{0}(a)\right|^{\boldsymbol{p}(a)}=\alpha$. Observe that $f_{0}(B)=f_{0}(|\boldsymbol{p}|)$. Let $B_{0} \subset B$ be such that $\left.f_{0}\right|_{B_{0}}$ is injective and $f_{0}\left(B_{0}\right)=f_{0}(B)$. We have

$$
\begin{aligned}
\alpha & \geq \prod_{\mu \in f_{0}(|\boldsymbol{p}|)}|\mu|^{\sup p\left(f_{0}^{-1}(\mu)\right)}=\prod_{\mu \in f_{0}\left(B_{0}\right)}|\mu|^{\sup p\left(f_{0}^{-1}(\mu)\right)} \\
& =\prod_{a \in B_{0}}\left|f_{0}(a)\right|^{\max \left\{\boldsymbol{p}(b): b \in B, f_{0}(b)=f_{0}(a)\right\}} \geq \prod_{a \in B}\left|f_{0}(a)\right|^{\boldsymbol{p}(a)}=\alpha .
\end{aligned}
$$

Property 4.5. $\log d_{G}^{\min }(\boldsymbol{p}, \cdot) \in \mathcal{P S H}(G)$ (cf. 2.6).

Proof. By 4.2(a), we only need to show that for any $f \in \mathcal{O}(G, E)$ the function $u_{f}(z):=\prod_{\mu \in f(G)}\left[m_{E}(\mu, f(z))\right]^{\sup p\left(f^{-1}(\mu)\right)}, z \in G$, is log-plurisubharmonic on $G$. The proof of 4.2 shows that $u_{f}=\inf _{M} v_{M}$, where $v_{M}$ is a log-plurisubharmonic function given by the formula

$$
v_{M}:=\prod_{\mu \in M}\left[m_{E}(\mu, f(z))\right]^{k_{f}(\mu)}
$$

and $M$ runs over a family of finite sets as in the proof of 4.2 . Observe that $v_{M_{1} \cup M_{2}} \leq \min \left\{v_{M_{1}}, v_{M_{2}}\right\}$. It remains to apply Lemma 2.9 .

PROPERTY 4.6. If $G_{k} \nearrow G$ and $\boldsymbol{p}_{k} \nearrow \boldsymbol{p}$, then

$$
d_{G_{k}}^{\min }\left(\boldsymbol{p}_{k}, z\right) \searrow d_{G}^{\min }(\boldsymbol{p}, z), \quad d_{G_{k}}^{\max }\left(\boldsymbol{p}_{k}, z\right) \searrow d_{G}^{\max }(\boldsymbol{p}, z), \quad z \in G .
$$

Proof. By $(\mathrm{H})$ and $(\mathrm{M})$ the sequences are monotone and for the limit functions $u$ we have $u \geq d_{G}^{\min }(\boldsymbol{p}, \cdot)\left(\right.$ resp. $\left.u \geq d_{G}^{\max }(\boldsymbol{p}, \cdot)\right)$. Fix a $z_{0} \in G$.

In the case of the minimal family suppose that $u\left(z_{0}\right)>\alpha>d_{G}^{\min }\left(G, z_{0}\right)$. Let $f_{k} \in \mathcal{O}\left(G_{k}, E\right)$ be such that $f_{k}\left(z_{0}\right)=0$ and

$$
\prod_{\mu \in f_{k}\left(G_{k}\right)}|\mu|^{\sup p_{k}\left(f_{k}^{-1}(\mu)\right)} \rightarrow u\left(z_{0}\right) .
$$

By a Montel argument we may assume that $f_{k} \rightarrow f_{0}$ locally uniformly in $G$ with $f_{0} \in \mathcal{O}(G, E), f_{0}\left(z_{0}\right)=0$. Since $\prod_{\mu \in f_{0}(G)}|\mu|^{\sup p\left(f_{0}^{-1}(\mu)\right)}<\alpha$, we can find a finite set $A \subset G$ such that $\left.f\right|_{A}$ is injective and $\prod_{a \in A}\left|f_{0}(a)\right|^{p(a)}<\alpha$. Consequently, $\prod_{a \in A}\left|f_{k}(a)\right|^{p_{k}(a)}<\alpha$ and $\left.f_{k}\right|_{A}$ is injective for $k \gg 1$. Finally, $\prod_{\mu \in f_{k}\left(G_{k}\right)}|\mu|^{\sup p_{k}\left(f_{k}^{-1}(\mu)\right)}<\alpha$ for $k \gg 1$; contradiction. 
In the case of the maximal family for any $a \in G$ and $\varepsilon>0$ there exists a $k(a, \varepsilon) \in \mathbb{N}$ such that $z_{0}, a \in G_{k}, \widetilde{k}_{G_{k}}^{*}\left(a, z_{0}\right) \leq \widetilde{k}_{G}^{*}\left(a, z_{0}\right)+\varepsilon$, and $\boldsymbol{p}_{k}(a) \geq$ $\boldsymbol{p}(a)-\varepsilon$ for $k \geq k(a, \varepsilon)$. Hence

$$
\begin{aligned}
\inf _{k \in \mathbb{N}} d_{G_{k}}^{\max }\left(\boldsymbol{p}_{k}, z_{0}\right) & =\inf _{k \in \mathbb{N}: a \in G_{k}}\left[\widetilde{k}_{G_{k}}^{*}\left(a, z_{0}\right)\right]^{p_{k}(a)} \\
& \leq \inf _{a \in G} \inf \left\{\left[\widetilde{k}_{G}^{*}\left(a, z_{0}\right)+\varepsilon\right]^{\boldsymbol{p}_{k}(a)}: 0<\varepsilon \ll 1, k \geq k(a, \varepsilon)\right\} \\
& \leq \inf _{a \in G} \inf \left\{\left[\widetilde{k}_{G}^{*}\left(a, z_{0}\right)+\varepsilon\right]^{p(a)-\varepsilon}: 0<\varepsilon \ll 1\right\}=d_{G}^{\max }\left(\boldsymbol{p}, z_{0}\right) .
\end{aligned}
$$

EXAMPLE 4.7. Let $G:=\left\{z \in \mathbb{C}^{n}:\left|z^{\alpha}\right|<1\right\}$, where $\alpha=\left(\alpha_{1}, \ldots, \alpha_{n}\right) \in$ $\mathbb{N}^{n}$ with $\alpha_{1}, \ldots, \alpha_{n}$ relatively prime. Then

$$
d_{G}^{\min }(\boldsymbol{p}, z)=d_{E}^{\min }\left(\boldsymbol{p}^{\prime}, z^{\alpha}\right)=\prod_{\mu \in E}\left[m_{E}\left(\mu, z^{\alpha}\right)\right]^{\boldsymbol{p}^{\prime}(\mu)}, \quad z \in G,
$$

where $\boldsymbol{p}^{\prime}(\lambda)=\sup \left\{\boldsymbol{p}(a): a^{\alpha}=\lambda\right\}$ for $\lambda \in E$, and $d_{E}^{\min }\left(\boldsymbol{p}^{\prime}, \cdot\right):=0$ if there exists a $\lambda_{0} \in E$ with $\boldsymbol{p}^{\prime}\left(\lambda_{0}\right)=\infty$.

Indeed, it is known that any function $f \in \mathcal{O}(G, E)$ has the form $f=g \circ \Phi$, where $\Phi(z):=z^{\alpha}$ and $g \in \mathcal{O}(E, E)$ (cf. [Jar-Pfl 1993, §4.4]. Thus

$$
\begin{aligned}
& d_{G}^{\min }(\boldsymbol{p}, z)=\sup \left\{\prod_{\mu \in g(\Phi(G))}\left[m_{E}(\mu, g(\Phi(z)))\right]^{\sup \boldsymbol{p}\left(\Phi^{-1}\left(g^{-1}(\mu)\right)\right)}: g \in \mathcal{O}(E, E)\right\} \\
& =\sup \left\{\prod_{\mu \in g(E)}\left[m_{E}(\mu, g(\Phi(z)))\right]^{\sup \boldsymbol{p}^{\prime}\left(g^{-1}(\mu)\right)}: g \in \mathcal{O}(E, E)\right\}=d_{E}^{\min }\left(\boldsymbol{p}^{\prime}, \Phi(z)\right) .
\end{aligned}
$$

5. Product property. Let $\underline{d}=\left(d_{G}\right)_{G}$ be a generalized holomorphically contractible family with integer-valued weights. We say that $\underline{d}$ has the product property if

$$
d_{G \times D}(A \times B,(z, w))=\max \left\{d_{G}(A, z), d_{D}(B, w)\right\}, \quad(z, w) \in G \times D,
$$

for any domains $G \subset \mathbb{C}^{n}, D \subset \mathbb{C}^{m}$ and for any sets $\emptyset \neq A \subset G$, $\emptyset \neq B \subset D$. Notice that the inequality " $\geq$ " follows from $(\mathrm{H})$ applied to the projections $G \times D \rightarrow G, G \times D \rightarrow D$. The definition applies to the standard holomorphically contractible families and means that

$$
d_{G \times D}((a, b),(z, w))=\max \left\{d_{G}(a, z), d_{D}(b, w)\right\}, \quad(a, b),(z, w) \in G \times D .
$$

It is well known that the families $\left(\widetilde{k}_{G}^{*}\right)_{G},\left(c_{G}^{*}\right)_{G},\left(g_{G}\right)_{G}$ have the product property (cf. [Jar-Pfl 1993, Ch. 9], [Edi 1997], [Edi 1999], [Edi 2001]).

Moreover, it is known that the higher order Möbius functions $\left(m_{G}^{(k)}\right)_{G}$ with $k \geq 2$ fail the product property (cf. [Jar-Pfl 1993, Ch. 9]).

Thus it is natural to ask whether the minimal and maximal families have the product property.

Proposition 5.1. The system $\left(d_{G}^{\max }\right)_{G}$ has the product property. 
Proof. Fix $\left(z_{0}, w_{0}\right) \in G \times D$ and $\varepsilon>0$. Let $(a, b) \in A \times B$ be such that $\widetilde{k}_{G}^{*}\left(a, z_{0}\right) \leq d_{G}^{\max }\left(A, z_{0}\right)+\varepsilon, \widetilde{k}_{D}^{*}\left(b, w_{0}\right) \leq d_{G}^{\max }\left(B, w_{0}\right)+\varepsilon$. Then using the product property for $\left(\widetilde{k}_{G}^{*}\right)_{G}$, we get

$$
\begin{aligned}
d_{G \times D}^{\max }\left(A \times B,\left(z_{0}, w_{0}\right)\right) & \leq \widetilde{k}_{G \times D}^{*}\left((a, b),\left(z_{0}, w_{0}\right)\right) \\
& =\max \left\{\widetilde{k}_{G}^{*}\left(a, z_{0}\right), \widetilde{k}_{D}^{*}\left(b, w_{0}\right)\right\} \\
& \leq \max \left\{d_{G}^{\max }\left(A, z_{0}\right), d_{D}^{\max }\left(B, w_{0}\right)\right\}+\varepsilon .
\end{aligned}
$$

We do not know whether the system $\left(d_{G}^{\mathrm{min}}\right)_{G}$ has the product property. So far we have been able to handle only the case where $\# B=1$ (see Proposition 5.3). Recall that $d_{G}^{\min }(A, \cdot)=m_{G}(A, \cdot)$ (Corollary 3.1(c)).

Proposition 5.2. Assume that for any $n \in \mathbb{N}$, the system $\left(m_{G}\right)_{G}$ has the following special product property:

$\left(\mathrm{P}_{0}\right)|\Psi(z, w)| \leq\left(\max _{G \times D}|\Psi|\right) \max \left\{m_{G}(A, z), m_{D}(B, w)\right\}, \quad(z, w) \in G \times D$,

where $G, D \subset \mathbb{C}^{n}$ are balls with respect to arbitrary $\mathbb{C}$-norms, $A \subset D, B \subset G$ are finite and non-empty, $\Psi(z, w):=\sum_{j=1}^{n} z_{j} w_{j}$, and $\left.\Psi\right|_{A \times B}=0$. Then the system $\left(m_{G}\right)_{G}$ has the product property $(\mathrm{P})$ in full generality. Moreover, if $\left(\mathrm{P}_{0}\right)$ holds with $\# B=1$, then $(\mathrm{P})$ holds with $\# B=1$.

Proof (cf. [Jar-Pfl 1993, the proof of Th. 9.5]). Fix arbitrary domains $G \subset \mathbb{C}^{n}, D \subset \mathbb{C}^{m}$, non-empty sets $A \subset G, B \subset G$, and $\left(z_{0}, w_{0}\right) \in G \times D$. We have to prove that for any $F \in \mathcal{O}(G \times D, E)$ with $\left.F\right|_{A \times B}=0$,

$$
\left|F\left(z_{0}, w_{0}\right)\right| \leq \max \left\{m_{G}\left(A, z_{0}\right), m_{D}\left(B, w_{0}\right)\right\} .
$$

By 2.12, we may assume that $A, B$ are finite.

Let $\left(G_{\nu}\right)_{\nu=1}^{\infty},\left(D_{\nu}\right)_{\nu=1}^{\infty}$ be sequences of relatively compact subdomains of $G$ and $D$, respectively, such that $A \cup\left\{z_{0}\right\} \subset G_{\nu} \nearrow G, B \cup\left\{w_{0}\right\} \subset D_{\nu} \nearrow D$. By 2.7, it suffices to show that

$$
\left|F\left(z_{0}, w_{0}\right)\right| \leq \max \left\{m_{G_{\nu}}\left(A, z_{0}\right), m_{D_{\nu}}\left(B, w_{0}\right)\right\}, \quad \nu \geq 1 .
$$

Fix a $\nu_{0} \in \mathbb{N}$ and let $G^{\prime}:=G_{\nu_{0}}, D^{\prime}:=D_{\nu_{0}}$. It is well known that $F$ may be approximated locally uniformly in $G \times D$ by functions of the form

$$
F_{s}(z, w)=\sum_{\mu=1}^{N_{s}} f_{s, \mu}(z) g_{s, \mu}(w), \quad(z, w) \in G \times D,
$$

where $f_{s, \mu} \in \mathcal{O}(G), g_{s, \mu} \in \mathcal{O}(D), s \geq 1, \mu=1, \ldots, N_{s}$. Notice that $F_{s} \rightarrow 0$ uniformly on $A \times B$. Using the Lagrange interpolation formula, we find polynomials $P_{s}: \mathbb{C}^{n} \times \mathbb{C}^{m} \rightarrow \mathbb{C}$ such that $\left.P_{s}\right|_{A \times B}=\left.F_{s}\right|_{A \times B}$ and $P_{s} \rightarrow 0$ locally uniformly in $\mathbb{C}^{n} \times \mathbb{C}^{m}$. The functions $\widehat{F}_{s}:=F_{s}-P_{s}, s \geq 1$, also have the form 
(**) and $\widehat{F}_{s} \rightarrow F$ locally uniformly in $G \times D$. Hence, without loss of generality, we may assume that $\left.F_{s}\right|_{A \times B}=0$ for $s \geq 1$. Let $m_{s}:=\max \left\{1,\left\|F_{s}\right\|_{G^{\prime} \times D^{\prime}}\right\}$ and $\widetilde{F}_{s}:=F_{s} / m_{s}, s \geq 1$. Note that $m_{s} \rightarrow 1$, and therefore $\widetilde{F}_{s} \rightarrow F$ uniformly on $G^{\prime} \times D^{\prime}$. Consequently, we may assume that $F_{s}\left(G^{\prime} \times D^{\prime}\right) \Subset E$ for $s \geq 1$.

It is enough to prove that

$$
\left|F_{s}\left(z_{0}, w_{0}\right)\right| \leq \max \left\{m_{G^{\prime}}\left(A, z_{0}\right), m_{D^{\prime}}\left(B, w_{0}\right)\right\}, \quad s \geq 1 .
$$

Fix an $s=s_{0} \in \mathbb{N}$ and let $N:=N_{s_{0}}, f_{\mu}:=f_{s_{0}, \mu}, g_{\mu}:=g_{s_{0}, \mu}, \mu=$ $1, \ldots, N$. Let $f:=\left(f_{1}, \ldots, f_{N}\right): G \rightarrow \mathbb{C}^{N}$ and $g:=\left(g_{1}, \ldots, g_{N}\right): D \rightarrow \mathbb{C}^{N}$. Put

$$
\begin{aligned}
& K:=\left\{\xi=\left(\xi_{1}, \ldots, \xi_{N}\right) \in \mathbb{C}^{N}:\right. \\
&\left.\left|\xi_{\mu}\right| \leq\left\|f_{\mu}\right\|_{G^{\prime}}, \mu=1, \ldots, N,|\Psi(\xi, g(w))| \leq 1, w \in D^{\prime}\right\} .
\end{aligned}
$$

It is clear that $K$ is an absolutely convex compact subset of $\mathbb{C}^{N}$ with $f\left(G^{\prime}\right) \subset K$. Let

$$
\begin{aligned}
L:=\left\{\eta=\left(\eta_{1}, \ldots, \eta_{N}\right)\right. & \in \mathbb{C}^{N}: \\
\left|\eta_{\mu}\right| & \left.\leq\left\|g_{\mu}\right\|_{D^{\prime}}, \mu=1, \ldots, N,|\Psi(\xi, \eta)| \leq 1, \xi \in K\right\} .
\end{aligned}
$$

Then again $L$ is an absolutely convex compact subset of $\mathbb{C}^{N}$, and moreover, $g\left(D^{\prime}\right) \subset L$.

Let $\left(W_{\sigma}\right)_{\sigma=1}^{\infty}$ (resp. $\left.\left(V_{\sigma}\right)_{\sigma=1}^{\infty}\right)$ be a sequence of absolutely convex bounded domains in $\mathbb{C}^{N}$ such that $W_{\sigma+1} \Subset W_{\sigma}$ and $W_{\sigma} \searrow K$ (resp. $V_{\sigma+1} \Subset V_{\sigma}$ and $\left.V_{\sigma} \searrow L\right)$. Put $M_{\sigma}:=\|\Psi\|_{W_{\sigma} \times V_{\sigma}}, \sigma \in \mathbb{N}$. By $\left(\mathrm{P}_{0}\right)$ and by the holomorphic contractibility applied to the mappings $f: G^{\prime} \rightarrow W_{\sigma}, g: D^{\prime} \rightarrow V_{\sigma}$ we have

$$
\begin{aligned}
\left|F_{s_{0}}\left(z_{0}, w_{0}\right)\right| & =\left|\Psi\left(f\left(z_{0}\right), g\left(w_{0}\right)\right)\right| \\
& \leq M_{\sigma} \max \left\{m_{W_{\sigma}}\left(f(A), f\left(z_{0}\right)\right), m_{V \sigma}\left(g(B), g\left(w_{0}\right)\right)\right\} \\
& \leq M_{\sigma} \max \left\{m_{G^{\prime}}\left(f^{-1}(f(A)), z_{0}\right), m_{D^{\prime}}\left(g^{-1}(g(B)), w_{0}\right)\right\} \\
& \leq M_{\sigma} \max \left\{m_{G^{\prime}}\left(A, z_{0}\right), m_{D^{\prime}}\left(B, w_{0}\right)\right\} .
\end{aligned}
$$

Letting $\sigma \rightarrow \infty$ we get the required result.

Proposition 5.3. The system $\left(m_{G}\right)_{G}$ has the product property $(\mathrm{P})$ whenever $\# B=1$, i.e. for any domains $G \subset \mathbb{C}^{n}, D \subset \mathbb{C}^{m}$, any set $A \subset G$, and any $b \in D$ we have

$$
m_{G \times D}(A \times\{b\},(z, w))=\max \left\{m_{G}(A, z), m_{D}(b, w)\right\}, \quad(z, w) \in G \times D .
$$

Proof. By Proposition 5.2, we only need to check (P) in the case where $D$ is a bounded convex domain, $A$ is finite, and $B=\{b\}$. Fix $\left(z_{0}, w_{0}\right) \in$ $G \times D$. Let $\varphi: E \rightarrow D$ be a holomorphic mapping such that $\varphi(0)=b$ and $\varphi\left(m_{D}\left(b, w_{0}\right)\right)=w_{0}$ (cf. [Jar-Pfl 1993, Ch. 8]). Consider the mapping $F: G \times E \rightarrow G \times D, F(z, \lambda):=(z, \varphi(\lambda))$. Then

$$
m_{G \times D}\left(A \times\{b\},\left(z_{0}, w_{0}\right)\right) \leq m_{G \times E}\left(A \times\{0\},\left(z_{0}, m_{G}\left(b, w_{0}\right)\right)\right) .
$$


Consequently, it suffices to show that

$$
m_{G \times E}\left(A \times\{0\},\left(z_{0}, \lambda\right)\right) \leq \max \left\{m_{G}\left(A, z_{0}\right),|\lambda|\right\}, \quad \lambda \in E .
$$

The case where $m_{G}\left(A, z_{0}\right)=0$ is elementary: for an $f \in \mathcal{O}(G \times E, E)$ with $\left.f\right|_{A \times\{0\}}=0$ we have $f\left(z_{0}, 0\right)=0$ and hence $\left|f\left(z_{0}, \lambda\right)\right| \leq|\lambda|$ for $\lambda \in E$ (by the Schwarz lemma). Thus, we may assume that $r:=m_{G}\left(A, z_{0}\right)>0$. First observe that it suffices to prove $(\dagger)$ on the circle $|\lambda|=r$. Indeed, if the inequality holds on that circle, then by the maximum principle for subharmonic functions (applied to the function $\left.m_{G \times E}\left(A \times\{0\},\left(z_{0}, \cdot\right)\right)\right)$ it holds for all $|\lambda| \leq r$. In the annulus $\{r<|\lambda|<1\}$ we apply the maximum principle to the subharmonic function $\lambda \mapsto|\lambda|^{-1} m_{G \times E}\left(A \times\{0\},\left(z_{0}, \lambda\right)\right)$.

Now fix a $\lambda_{0} \in E$ with $\left|\lambda_{0}\right|=r$. Let $f$ be an extremal function for $m_{G}\left(A, z_{0}\right)$ with $\left.f\right|_{A}=0$ and $f\left(z_{0}\right)=\lambda_{0}$. Consider $F: G \rightarrow G \times E, F(z):=$ $(z, f(z))$. Then

$$
m_{G}\left(A \times\{0\},\left(z_{0}, \lambda_{0}\right)\right) \leq m_{G}\left(A, z_{0}\right)=\max \left\{m_{G}\left(A, z_{0}\right),\left|\lambda_{0}\right|\right\},
$$

which completes the proof.

Acknowledgements. The authors are indebted to Professor Włodzimierz Zwonek for his valuable comments on the paper.

\section{References}

[Car-Wie 2003] M. Carlehed et J. Wiegerinck, Le cône des fonctions plurisousharmoniques négatives et une conjecture de Coman, Ann. Polon. Math. 80 (2003), 93-108.

[Com 2000] D. Coman, The pluricomplex Green function with two poles of the unit ball of $\mathbb{C}^{n}$, Pacific J. Math. 194 (2000), 257-283.

[Edi 1997] A. Edigarian, On the product property of the pluricomplex Green function, Proc. Amer. Math. Soc. 125 (1997), 2855-2858.

[Edi 1999] —, Remarks on the pluricomplex Green function, Univ. Iagel. Acta Math. 37 (1999), 159-164.

[Edi 2001] -, On the product property of the pluricomplex Green function, II, Bull. Polish Acad. Sci. Math. 49 (2001), 389-394.

[Edi 2002] _, Analytic discs method in complex analysis, Dissertationes Math. 402 (2002).

[Edi-Zwo 1998] A. Edigarian and W. Zwonek, Invariance of the pluricomplex Green function under proper mappings with applications, Complex Variables 35 (1998), 367-380.

[Jar-Pfl 1993] M. Jarnicki and P. Pflug, Invariant Distances and Metrics in Complex Analysis, de Gruyter Exp. Math. 9, de Gruyter, 1993.

[Lár-Sig 1998] F. Lárusson and R. Sigurdsson, Plurisubharmonic functions and analytic discs on manifolds, J. Reine Angew. Math. 501 (1998), 1-39.

[Lel 1989] P. Lelong, Fonction de Green pluricomplexe et lemme de Schwarz dans les espaces de Banach, J. Math. Pures Appl. 68 (1989), 319-347.

[Zwo 2000a] W. Zwonek, Regularity properties of the Azukawa metric, J. Math. Soc. Japan 52 (2000), 899-914. 
[Zwo 2000b] W. Zwonek, Completeness, Reinhardt domains and the method of complex geodesics in the theory of invariant functions, Dissertationes Math. 388 (2000).

Institute of Mathematics

Jagiellonian University

Reymonta 4

30-059 Kraków, Poland

E-mail: jarnicki@im.uj.edu.pl

wmj@im.uj.edu.pl
Fachbereich Mathematik

Carl von Ossietzky Universität Oldenburg

Postfach 2503

D-26111 Oldenburg, Germany

E-mail: pflug@mathematik.uni-oldenburg.de

Reçu par la Rédaction le 25.11.2002 